\title{
Especies de dinoflagelados atecados (Dinophyta) de la costa de Chiapas, sur del Pacífico mexicano
}

\author{
Species of athecate dinoflagellates (Dinophyta) from coasts of Chiapas, southern Mexican Pacific
}

\section{Ebodio Maciel-Baltazar¹ y David U. Hernández-Becerril ${ }^{2}$}

\author{
${ }^{1}$ Laboratorio Estatal de Salud Pública, Instituto de Salud del Estado de Chiapas, Blvd. Salomón Gonzales Blanco 3452, Tuxtla \\ Gutiérrez, 29040, Chiapas, México \\ ${ }^{2}$ Instituto de Ciencias del Mar y Limnología, Universidad Nacional Autónoma de México, Apdo. postal 70-305, Ciudad \\ Universitaria, Coyoacán, 04510, México, D.F., México.dhernand@cmarl.unam.mx
}

\begin{abstract}
Athecate dinoflagellates have been poorly studied in the plankton of Mexican marine waters, mainly because of their fragility, as they may become deformed using nets and strong fixatives. However, their biodiversity and ecological role might be important in the planktonic realm. As part of routine phytoplankton monitoring in the Chiapas coasts, Mexico, in the southern Mexican Pacific, samples were obtained during 2009 by net ( $20 \mu \mathrm{m}$ mesh) in vertical hauls (up to $15 \mathrm{~m}$ ), fixed with Lugol's solution and studied by light microscope (bright field and phase contrast). Athecate dinoflagellates species were identified using morphological characters such as shape and size, nucleus position, chloroplasts number and position, and particular characters ('arms', 'carina', etc.). Twenty-seven species were documented to be present in the study area, with 3 species considered to be new records for the Mexican Pacific Ocean: Cochlodinium pulchellum, Karenia bicuneiformis ( $=$ K. bidigitata) and K. papilionacea. Few species studied here have historically been reported as 'bloomforming' species in other parts of the Mexican Pacific. It is clear that more studies should be systematically done to assess the present biodiversity of these dinoflagellates groups in Mexican waters.
\end{abstract}

Key words: Dinoflagellates, phytoplankton, morphology, new records, Mexican Pacific

Resumen.- Los dinoflagelados atecados han sido poco estudiados en las costas mexicanas, debido principalmente a su fragilidad, ya que se deforman por la acción de los fijadores fuertes o durante su captura con redes. Sin embargo, tanto su biodiversidad como su papel ecológico pueden ser muy importantes en el ambiente planctónico. Como parte del monitoreo en las costas de Chiapas, México, durante el 2009 se analizaron muestras recolectadas con red de $20 \mu \mathrm{m}$ de malla, en arrastres verticales desde una profundidad de $15 \mathrm{~m}$, las muestras fueron fijadas con solución de Lugol y estudiadas con microscopia de luz (técnicas de campo claro y contraste de fases). Las especies fueron identificadas usando caracteres morfológicos como son forma, tamaño, posición del núcleo, número y posición de cloroplastos y caracteres específicos (brazos, carena, etc.). En este trabajo se describen e ilustran 27 especies, de las cuales 3 son nuevos registros para el Pacífico mexicano: Cochlodinium pulchellum, Karenia bicuneiformis (= K. bidigitata) y K. papilionacea. Algunas especies han sido reportadas anteriormente por formar proliferaciones importantes en otras zonas del Pacífico mexicano. El inventario aún es incompleto y se requieren más estudios que permitan conocer la biodiversidad de este grupo de dinoflagelados en aguas mexicanas.

Palabras clave: Dinoflagelados, fitoplancton, morfología, nuevos registros, Pacífico mexicano

\section{INTRODUCCIÓN}

En el fitoplancton marino los dinoflagelados (Dinophyta o Dinoflagellata) son el segundo grupo de protistas por su importancia cuantitativa, biomasa, diversidad y distribución, solo después de las diatomeas. En términos de diversidad, se estima que existen actualmente cerca de 2000 especies (Taylor et al. 2008), aunque se han listado 1.555 dinoflagelados marinos de vida libre (Gómez 2005). En el Pacífico mexicano se hizo una recopilación bibliográfica indicando la existencia de 605 especies de dinoflagelados, con 199 especies registradas en el golfo de Tehuantepec y sólo 34 especies en costas de Chiapas
(Okolodkov \& Gárate-Lizárraga 2006), mientras que Meave del Castillo \& Hernández-Becerril (1998) listaron 102 taxa de dinoflagelados en el golfo de Tehuantepec, y más recientemente, en la zona costera de Acapulco, Meave del Castillo et al. (2012) encontraron 347 taxa de dinoflagelados, con 32 taxa de dinoflagelados desnudos de nuevo registro.

Los dinoflagelados atecados (o desnudos) no son un grupo natural (monofilético), pero todos muestran en general una pared frágil y placas muy finas, lo cual es 
difícil de observar con microscopia de luz: las diferentes especies se distinguen por caracteres morfológicos, tales como el tamaño y forma general, la morfología, posición y desplazamiento del cíngulo y del sulcus, presencia o ausencia de cloroplastos y pirenoides, forma y posición del núcleo, forma del surco apical (si está presente) y posibles estructuras en la superficie, tales como estrías longitudinales. La observación de otros rasgos, como estigmas y apéndices, es importante, aunque muchos miembros de este grupo, se deforman o se desintegran en el momento de fijar la muestra lo que los hace poco reconocibles durante el proceso de determinación taxonómica, razón por la cual en una muestra de plancton, aparecen como especies relativamente raras o ausentes. Sin embargo, algunas son lo suficientemente robustas y se mantienen en buenas condiciones durante periodos de tiempo prolongados, de tal forma que muchas de las descripciones se han basado en material fijado. No obstante, por su carácter delicado no mantienen la morfología tras la fijación, razón por la que muchas especies han sido pobremente descritas y que probablemente no podrán ser nunca identificadas (Larsen 2002).

En este trabajo se ha estudiado la morfología de los dinoflagelados atecados, presentes en muestras costeras de Chiapas, en el sur del Pacífico mexicano, a través de microscopía de luz y siguiendo protocolos de análisis particulares para este grupo, con el propósito de conocer su biodiversidad y potencial contribución a la biomasa y productividad.

\section{Materiales y MÉtodos}

La zona de estudio se localiza dentro del golfo de Tehuantepec (GT) (Fig. 1), que se encuentra dividida en regiones condicionadas por la variabilidad de los vientos (Trasviña \& Burton 1997), dentro de la región hidrográfica oriental, a la cual pertenece la costa de Chiapas, de Paredón a la frontera con Guatemala, caracterizada por ser la que menos está influida por los nortes 'Tehuanos' y presentar una superficie termohalina menos salina y más cálida que el área adyacente (Monreal \& Salas de León 1998). Existen 2 estaciones bien diferenciadas, la seca de octubre-abril, cuando las corrientes fluyen paralelas a la costa, influidas por la corriente de Costa Rica, y la de lluvias mayo-septiembre, cuando ocurre la presencia de huracanes y tormentas tropicales.

Se analizaron 24 muestras, obtenidas durante enerodiciembre, 2009, mediante arrastres verticales de red (45 $\mathrm{cm}$ de diámetro y $20 \mu \mathrm{m}$ de abertura de malla), desde $15 \mathrm{~m}$ de profundidad, procedentes de 3 estaciones costeras fijas (E-1, E-2 y E-3, Fig. 1). El material fue puesto en frascos de $250 \mathrm{ml}$ de capacidad, para ser fijados con una solución de Lugol ácido. En lo posible, las muestras fueron observadas inmediatamente o en un plazo no mayor a un mes, con un microscopio invertido Axiostar 25 Carl Zeiss ${ }^{\circledR}$, con objetivo de 40X, utilizando técnicas de campo claro y contraste de fases para el reconocimiento de las especies. Las microfotografías fueron tomadas con una cámara digital Kodak ${ }^{\circledR}$ M853 de 8.2 megapixeles y las medidas de los organismos se obtuvieron con un analizador de

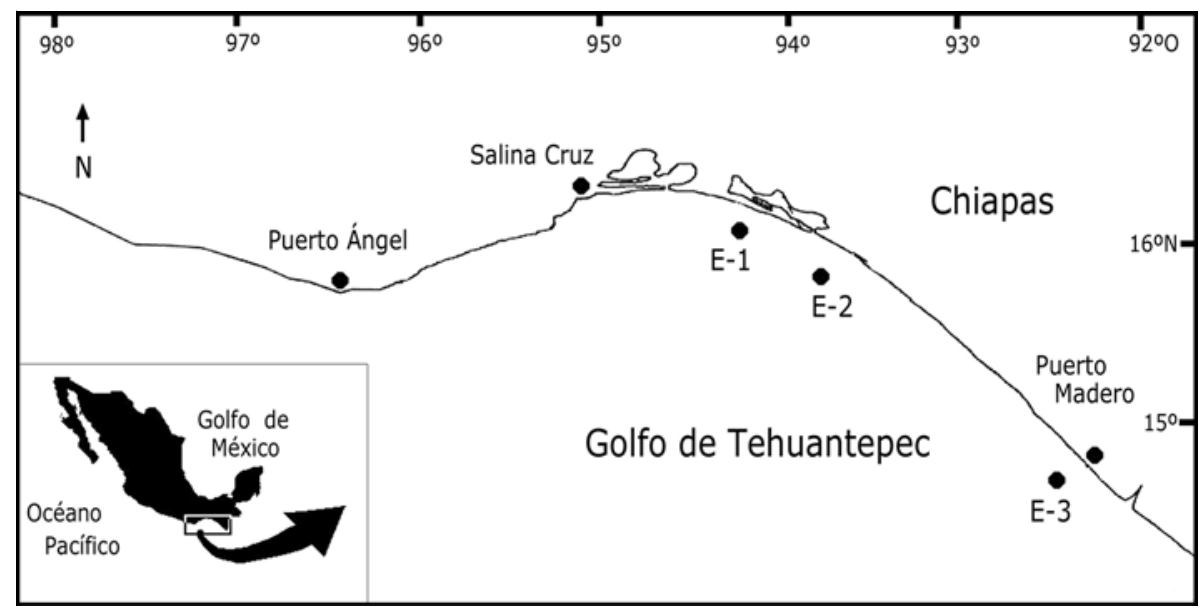

Figura 1. Localización del área de estudio en el Golfo de Tehuantepec y los puntos de recolecta de fitoplancton (E1, E-2 y E-3) / Study area in the Gulf of Tehuantepec, with the phytoplankton sampling points (E1, E-2 and E-3) 
imagen AXIOVISION ${ }^{\mathrm{TM}}$ v. 4.8.1 y fueron las siguientes: longitud total (lt), transdiámetro (td) y diámetro (d). La posición sistemática sigue las propuestas de Fensome et al. (1993).

\section{Resultados}

Se presenta una breve descripción de las especies estudiadas, en particular los caracteres distintivos, así como su respectiva ilustración.

\section{División Dinoflagellata (Bütschli) Fensome}

\section{Clase Dinophyceae Pascher}

\section{Orden Gymnodiniales LemmermanN}

FAMiLIa GyMnOdiniaceae LANKESTER

\section{Género Akashiwo G. Hansen et Moestrup}

\section{Akashiwo sanguinea (Hirasaka) G. Hansen et Moestrup} (Fig. 2A)

Sinónimos: Gymnodinium sanguineum Hirasaka; G. splendens Lebour; G. nelsonii Martin

Referencias: Hallegraeff 2002, p. 42, fig. 10B; Avancini et al. 2006, p. 265, figs. A-C; Alonso-Rodríguez et al. 2008, p. 116-117.

Especie pleomórfica con mayor variación en el hipocono, generalmente aplanada dorsoventralmente, epicono de redondeado a cónico, hipocono bilobulado, el núcleo se encuentra situado ligeramente anterior en la parte media de la célula.

Dimensiones: $1 \mathrm{t}=40-80 \mu \mathrm{m}, \mathrm{td}=35-60 \mu \mathrm{m}$.

Distribución general: cosmopolita, se presenta en aguas estuarinas y costeras de climas templados a tropicales.

Distribución en México: frecuente en el Pacífico mexicano, del golfo de Calfornia (GC), hasta las costas de Oaxaca.

Akashiwo sanguinea es una especie catalogada como nociva, se reporta como formadora de mareas rojas en el golfo de California (Alonso-Rodriguez et al. 2008) y en Costa Rica (Vargas-Montero et al. 2008). En las costas de Chiapas es una especie frecuente en los muestreos, sin embargo no existen reportes de florecimientos.

\section{Género Amphidinium Claparède et LaChManN}

Amphidinium cf. operculatum Claparède et Lachmann (Fig. 2B)
Referencias: Jørgensen et al. 2004, p. 7, fig. 2C-D; Murray et al. 2004, p. 368, fig. 1A-F.

Célula de forma oval en vista ventral y aplanada dorsoventralmente, se distingue por tener un epicono pequeño en forma de 'dedo' dirigido hacia la izquierda. Núcleo ovoide en posición posterior. Las células están cubiertas por una pared membranosa que se distorsiona durante la fijación, lo que impidió asegurar la identificación.

Dimensiones: $1 \mathrm{t}=26 \mu \mathrm{m}, \mathrm{td}=18 \mu \mathrm{m}$.

Distribución general: la localidad tipo corresponde a las aguas frías de Noruega, se ha reportado en aguas tropicales costeras y estuarinas.

Distribución en México: reportada para el GC y en este estudio se menciona la ampliación de ámbito para el golfo de Tehuantepec (GT).

Amphidinium operculatum es una especie reportada como productora de sustancias hemolíticas (ictiotoxinas) igual que A. carterae. Puede existir alguna confusión taxonómica entre estas 2 especies al tener una gran similitud de forma y tamaño, pero se diferencian por la disposición radial de los cloroplastos y el tamaño algo mayor de la primera. El único reporte de esta especie para México, en concreto para el GC, es de Núñez-Vázquez (2005), citado por Okolodkov \& Gárate-Lizárraga (2006).

\section{GÉNERo CoCHLODINIUM SCHÜTT}

\section{Cochlodinium helicoides Lebour (Fig. 2C)}

Referencias: Dodge 1982, p. 76, fig. 8G; Gárate-Lizárraga et al. 2011, p. 496, fig. 2A-C.

Célula de forma irregular, el extremo anterior es angosto mientras que el posterior es amplio y se extiende formando una especie de lóbulo como si fuera un colgajo dando la apariencia de una excavación, el cíngulo presenta una espiral de 1,5 vueltas, el núcleo es central y cloroplastos amarillos, algunos especímenes se encuentran rodeados por una capa hialina, estructura de un quiste temporal.

Dimensiones: $1 \mathrm{t}=29-54 \mu \mathrm{m}, \mathrm{td}=24-30 \mu \mathrm{m}$.

Distribución: reportada en las Islas Británicas, mar Mediterráneo y el océano Pacífico.

Distribución en México: se ha reportado para el GC. En este estudio se reconoce la ampliación de ámbito de $C$. helicoides para la costa de Chiapas. 


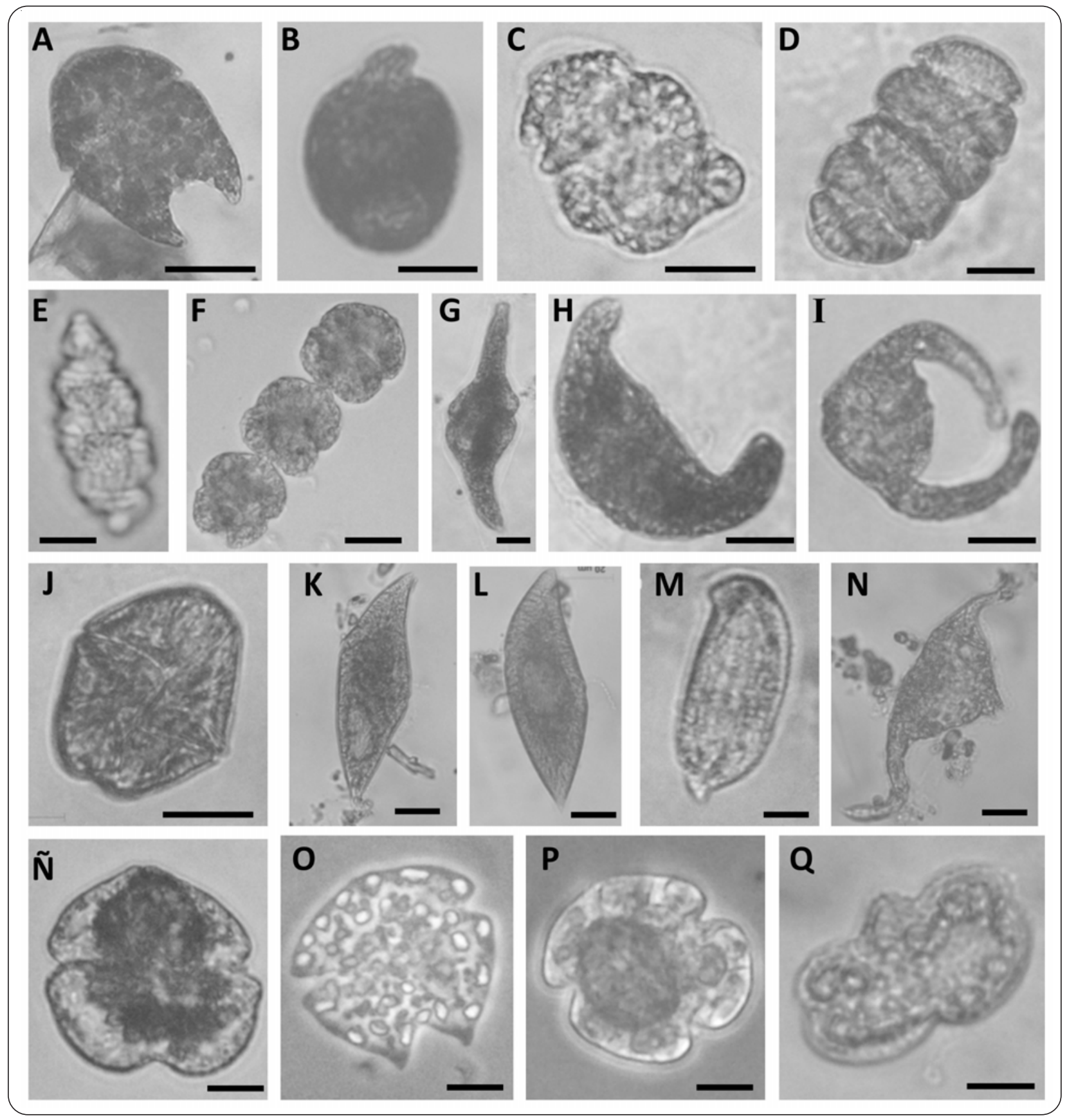

Figura 2. Especies de dinoflagelados atecados de costas de Chiapas, México, microscopía de luz. A) Akashiwo sanguinea, B) Amphidinium cf. operculatum, C) Cochlodinium helicoides, D) Célula viva de Cochlodinium polykrikoides, E) Cochlodinium pulchellum, F) Gymnodinium catenatum (cadena de 3 células fijadas), G-I) Gyrodinum falcatum, morfotipo típico y otros 2 morfotipos (estadios de ciclo de vida), respectivamente; (I) también conocido como Pseliodinium vaubanii, J) Vista ventral de Gyrodinium instriatum, K-L) Dos especímenes distintos de Gyrodinium spirale, M) Torodinium teredo, N) Gynogonadinium aequatoriale, N) Karenia asterichroma, 0) Karenia bicuneiformis, P) Karenia mikimotoi, Q) Karenia papilionacea. Escalas en Figs. B y M-Q representan $\mathbf{1 0} \boldsymbol{\mu m}$ y las demás $\mathbf{2 0} \boldsymbol{\mu m} /$ Species of athecate dinoflagellates from the coasts of Chiapas, Mexico, light microscopy. A) Akashiwo sanguinea, B) Amphidinium cf. operculatum, C) Cochlodinium helicoides, D) Live cell of Cochlodinium poyikrikoides, E) Cochlodinium pulchellum, F) Gymnodinium catenatum (chain of 3 fixed cells), G-I) Gyrodinum falcatum, typical morphotype and other 2 morphotypes (life cycle stages), respectively; (I) is also recongnized as Pseliodinium vaubanii, J) Ventral view of Gyrodinium instriatum, K-L) Two different specimens of Gyrodinium spirale, M) Torodinium teredo, N) Gynogonadinium aequatoriale, Ñ) Karenia asterichroma, O) Karenia bicuneiformis, P) Karenia mikimotoi, Q) Karenia papilionacea. Scales in Figs. B and M-Q represent $10 \mu \mathrm{m}$, the rest represent $20 \mu \mathrm{m}$ 
Cochlodinium polykrikoides Margalef (Fig. 2D)

Referencias: Steidinger \& Tangen 1997, p. 446, plate 17; Iwataki et al. 2007, p. 234, figs. 10-17; Matsuoka et al. 2008, p. 262, fig. 1A-F.

Células más largas que anchas, epicono de tipo cónico, hipocono bilobulado, cíngulo profundo rodea la célula entre 1,8-1,9 veces, el sulcus se encuentra situado por debajo de éste, presenta en vista dorsal un estigma de color rojo en la parte posterior del epicono, el núcleo se encuentra localizado anteriormente, se pueden presentar cadenas de 2, 4 y raramente 8 células.

Dimensiones: células individuales $1 \mathrm{t}=56-60 \mu \mathrm{m}, \mathrm{td}=39$ $42 \mu \mathrm{m}$, en la colonia de 2 células $1 \mathrm{t}=36-40 \mu \mathrm{m}, \mathrm{td}=32-35$ $\mu \mathrm{m}$.

Distribución general: cosmopolita, se encuentra en aguas templadas y cálidas.

Distribución en México: se ha reportado a lo largo de la costa del Pacífico, desde Colima al GC; en el presente estudio constituye la primer mención para el GT.

Se reporta por primera vez la presencia de $C$. polykrikoides para la región del GT, la distribución de esta especie ha sido registrada en el GC (Gárate-Lizárraga et al. 2004), hasta este estudio no existen reportes de $C$. polykrikoides para el GT, pero hay referencias de la especie para Costa Rica (Vargas-Montero et al. 2008). En el estado de Chiapas se han presentado florecimientos no reportados de C. polykrikoides desde el 2007. Si bien C. polykrikoides, no es catalogada como especie productora de toxinas que afectan la salud humana, sí es conocida como generadora de sustancias reactivas al oxígeno (aniones de superóxidos, hidroxilos y peróxido de hidrogeno), ROS por sus siglas en inglés, que afectan la transferencia de oxígeno en las branquias de los peces produciéndoles la muerte por asfixia.

\section{Cochlodinium pulchellum Lebour (Fig. 2E)}

Referencias: Dodge 1982, p. 78, fig. 8F.

Célula pequeña presenta el cingulum en forma de espiral que da aproximadamente 3 giros en forma descendente el epicono tiene forma cónica en tanto que el hipocono termina de forma más redondeada, el núcleo se encuentra en el tercio inferior del soma.

Dimensiones: $1 \mathrm{t}=36 \mu \mathrm{m}, \mathrm{td}=13,5 \mu \mathrm{m}$.

Distribución: inicialmente reportada para las Islas Británicas, el mar Mediterráneo y el océano Pacífico.
Distribución en México: primer registro de Cochlodinium pulchellum para el Pacífico mexicano.

Género Gymnodinium Stein emend. G. Hansen et Moestrup

\section{Gymnodinium catenatum Graham (Fig. 2F)}

Referencias: Licea et al. 1995 p. 26, lám. 7, fig. 8; Steidinger \& Tangen 1997, p. 447, plate 18; Alonso-Rodríguez et al. 2008, p. 118-119.

Células muy variables en formas y tamaño, en material fijado se observaron desde subesféricas bicónicas a cuadrangulares, forman cadenas hasta de 64 células. El cingulum describe una espiral descendente que puede estar desplazada hasta $1 / 5$ de la longitud celular, el sulcus se extiende desde el antápice hasta el ápice, poseen numerosos cloroplastos con pirenoides muy conspicuos.

Dimensiones: $1 \mathrm{t}=34-65 \mu \mathrm{m}, \mathrm{td}=27-43 \mu \mathrm{m}$.

Distribución: aparentemente cosmopolita en aguas tropicales.

Distribución en México: se reporta en todo el Pacífico mexicano.

A partir de su descripción original en el GC, ha sido reportada en todo el mundo excepto las regiones polares como formadora de florecimientos tóxicos, ya que es el único dinoflagelado desnudo que produce toxinas paralizantes del tipo de las saxitoxinas.

Género Gyrodinium Kofoid et SweZy emend. G. Hansen et Moestrup

\section{Gyrodinium falcatum Kofoid et Swezy (Fig. 2G-I)}

Sinónimos: Gyrodinium caudatum Kofoid et Swezy (Fig. 2H); Pseliodinium vaubanii Sournia (Fig. 2I)

Referencias: Konovalova 2003, p. 169, figs. 1-9; GárateLizárraga \& Verdugo-Díaz 2007, p. 41, lám. 1, fig. 8; Gómez 2007b, p. 274, figs. 3, 16, 17; Gárate-Lizárraga et al. 2010, p. 54, figs. 2-21.

Presenta formas fusiformes y ovaladas, con una extensión en el epicono y otra en el hipocono, durante los diferentes estadios de vida, estas extensiones y la apariencia del cuerpo varían en cuanto a longitud y forma celular, algunos ejemplares presentan el cuerpo más redondeado con extensiones más o menos cortas.

Dimensiones: $1 \mathrm{t}=119,6-164,3 \mu \mathrm{m}, \mathrm{td}=33,2-42,5 \mu \mathrm{m}$.

Distribución: de muy amplia distribución en aguas 
templadas y cálidas, se ha reportado para Japón, el Mediterráneo, el suroeste de Inglaterra, el golfo de México, al noroeste de Madagascar, en las costas de Brasil y el Pacífico sur.

Distribución en México: amplia distribución en el Pacífico Mexicano, se ha registrado tanto en el GC como en el GT, sin embargo, los registros de esta especie son escasos.

Gyrodinum falcatum ha sido citada previamente por Meave del Castillo \& Hernández-Becerril (1998) para el GT, Gárate-Lizárraga et al. (2010) y Gárate-Lizárraga \& Verdugo-Díaz (2007) la registraron en el GC. Konovalova (2003) mencionó que existen 9 estadios de vida de $G$. falcatum: los morfotipos en este estudio son similares a los reportados por Gárate-Lizárraga et al. (2010), incluyendo células vegetativas en condiciones 'normales' (Fig. 2G), morfotipos más cercanos a G. caudatum (Fig. $2 \mathrm{H})$ y el estadio 5 , considerado el estadio final del desarrollo vegetativo del organismo (Konovalova 2003), el cual fue descrito por Sournia (1972) como Pseliodinium vaubanii (Fig. 2I).

Gyrodinium instriatum Freudenthal et Lee (Fig. 2J)

Referencias: Uchida et al. 1996, p. 120, figs. 1-7; Steidinger \& Tangen 1997, p. 452, plate 19.

Se diferencia de las especies de Gymnodinium por el agudo desplazamiento del cingulum a más de un tercio de la longitud del cuerpo formando una ' $Z$ ', la célula tiene forma dorsalmente comprimida, epicono convexo a ligeramente cóncavo, hipocono bilobulado con el sulcus extendido hacia la izquierda del hipocono y no presenta ranura apical. Muestra un núcleo esférico en el epicono.

Dimensiones $1 \mathrm{t}=48,6 \mu \mathrm{m}, \mathrm{td}=40 \mu \mathrm{m}$.

Distribución: cosmopolita, común en aguas templadas y tropicales,

Distribución en México: reportada desde el GC hasta el GT.

Gyrodinium instriatum ha sido reportada para el norte del Pacífico mexicano (Alonso-Rodríguez et al. 2008) y para las costas de Costa Rica (Vargas-Montero et al. 2008), como especie responsable de florecimientos, pero no había sido reportada para la zona del GT. No se conocen muchos registros de esta especie, los mencionados se han dado en el marco de investigaciones por florecimientos algales principalmente en ambientes mixohalinos.
Gyrodinium spirale (Bergh) Kofoid et Swezy (Figs. 2K, 2L)

Sinónimo: Gymnodinium spirale Bergh

Referencias: Steidinger \& Tangen 1997, p. 453, plate 20; Gárate-Lizárraga et al. 2009, p. 23, figs. 25, 26.

Célula de forma fusiforme, cingulum característico, descendente hacia la izquierda en espiral, desplazado más de 0,2 veces la longitud total del cuerpo. El núcleo está situado normalmente cerca del centro de la célula, superficie lisa o con estrías longitudinales.

Dimensiones $1 \mathrm{t}=96,5 \mu \mathrm{m}, \mathrm{td}=32,5 \mu \mathrm{m}$.

Distribución: cosmopolita de aguas costeras y estuarios subtropicales.

Distribución en México: la especie ha sido reportada a lo largo de la costa del Pacífico mexicano.

Es una especie reconocida por producir florecimientos en el GC, específicamente Bahía de la Paz (Gárate-Lizárraga et al. 2009).

\section{Género Torodinium Kofoid et Swezy}

Torodinium teredo (Pouchet) Kofoid et Swezy (Fig. 2M)

Sinónimo: Torodinium robustum Kofoid et Swezy.

Referencia: Steidinger \& Tangen 1997, p. 456, plate 20; Gómez 2009, p. 132, fig. 2A.

Presenta un epicono que ocupa 4/5 partes del largo de la célula, el cíngulo posterior $\mathrm{y}$ un hipocono extremadamente reducido en forma de cono invertido, el sulco se extiende a lo largo del epicono, dependiendo de la vista se puede observar una proyección lateral cerca de la punta como un pico de ave, presenta bandas longitudinales pigmentadas, el núcleo se puede distinguir como una región pálida que se extiende longitudinalmente en el centro del epicono.

Dimensiones: $1 \mathrm{t}=47,5-71,4 \mu \mathrm{m}, \mathrm{td}=20,4-29,4 \mu \mathrm{m}$.

Distribución: amplia distribución en aguas templadas y tropicales.

Distribución en México: Torodinium teredo ha sido encontrada recientemente en aguas costeras de Acapulco, costa Pacífica mexicana (Meave del Castillo et al. 2012). Okolodkov \& Gárate-Lizárraga (2006) discutieron la distribución de otra especie del género Torodinium, $T$. robustum Kofoid et Swezy, desde el GC hasta las costas 
de Guerrero. Gómez (2005) la cita como un sinónimo de $T$. teredo, por lo que será conveniente diferenciar las 2 especies y conocer su distribución más precisamente.

\section{GÉNERO GYNOGONADINIUM GÓMEZ}

\section{Gynogonadinium aequatoriale Gómez (Fig. 2N)}

Referencia: Gómez 2007a, p. 12, figs. 1-4.

La célula es lateralmente comprimida, por lo que la vista ventral es difícil de apreciar, en vista lateral el soma presenta una forma triangular característica, el cingulum en posición media, en tanto el núcleo es grande con forma elipsoidal y perpendicular al cingulum, de la región ventral parten 2 extensiones y relativamente del mismo tamaño que el cuerpo de la célula, la extensión apical termina en forma esférica.

Dimensiones: $1 \mathrm{t}=102 \mu \mathrm{m}, \mathrm{td}=25 \mu \mathrm{m}$.

Distribución: reportada únicamente en la localidad tipo que corresponde al océano Pacífico ecuatorial.

Distribución en México: recientemente registrada en el Pacífico mexicano, particularmente en Acapulco (Meave del Castillo et al. 2012).

Familia Kareniaceae Bergholtz, Daugbjerg, Moestrup et Fernández-Tejedor

\section{Género Karenia G. Hansen et Moestrup}

Karenia asterichroma De Salas, Bolch et Hallegraeff (Fig. 2N)

Referencias: De Salas et al. 2004, p. 627, figs. 5-7.

Su morfología es similar a K. mikimotoi y K. brevis, comprimida dorsoventralmente, epicono cónico con una cresta homologa a la carena y un reborde ventral como K. mikimotoi, canal apical recto, cíngulo localizado en la mitad de la célula, cloroplastos elongados que forman una especie de estrella que llegan hasta el hipocono, sulcus profundo, núcleo redondo situado en el centro del epicono.

Dimensiones: $1 \mathrm{t}=32 \mu \mathrm{m}, \mathrm{td}=20 \mu \mathrm{m}$.

Distribución: reportada por De Salas et al. (2004) para Tasmania y Australia.

Distribución en México: esta especie fue recientemente encontrada en Acapulco, aguas del Pacífico mexicano (Meave del Castillo et al. 2012).
Karenia bicuneiformis Botes, Sym et Pitcher (Fig. 2O)

Sinónimo: Karenia bidigitata Haywood et Steidinger

Referencias: Botes et al. 2003, p. 566, figs. 10-19; Haywood et al. 2004, p. 172, fig. 5; Gómez et al. 2005a, p. 269, fig. 22.

Presenta el epicono cónico, algunas veces redondeado no presenta carena, el núcleo es ovalado y ocupa la parte de la izquierda del hipocono, el cual está dividido en 2 extensiones con extremidades acentuadas en forma de ' $W$ ' que excede la longitud del epicono, muestra numerosos cloroplastos distribuidos a través de toda la célula.

Dimensiones: $1 \mathrm{t}=35-38 \mu \mathrm{m}, \mathrm{td}=32-34 \mu \mathrm{m}$.

Distribución: reportada originalmente para Sudáfrica, posteriormente ha sido mencionada en reportes de Nueva Zelanda y el Pacífico Sur.

Distribución en México: primer registro para el Pacífico mexicano.

Karenia mikimotoi (Miyake et Kominami ex Oda) G. Hansen et Moestrup (Fig. 2P)

Referencias: Hallegraeff 2002, p. 36, fig. 9C; Haywood et al. 2004, p. 167, fig. 1 c-I; Steidinger et al. 2008, p. 275, fig. 3.

Célula pequeña, ovalada a redonda, epicono cóncavo es más corto que el hipocono. El cíngulo es descendente y desplazado, carece de una carena apical, presenta cloroplastos dispuestos de forma irregular. El núcleo es elipsoidal a oblongo y está localizado a lo largo del lado izquierdo, extendiéndose desde el hipocono hasta el área del cíngulo o dentro del epicono.

Dimensiones: promedio $1 \mathrm{t}=32 \mu \mathrm{m}, \mathrm{td}=20 \mu \mathrm{m}$.

Distribución: Registrada para todo el mundo en aguas frías a tropicales.

Distribución en México: ha sido reportada para el GC, en este estudio documentamos la ampliación de ámbito de la especie para el GT.

En muchos de los reportes, ha sido confundida con especies similares, las cuales recientemente han sido descritas como nuevas especies, consecuentemente la verdadera distribución de K. mikimotoi debe ser revaluada. 
Karenia papilionacea Haywood et Steidinger (Fig. 2Q)

Referencias: Haywood et al. 2004, p. 167, fig. 3; Gómez et al. 2005a, p. 269, figs. 19, 21; Steidinger et al. 2008, p. 273, fig. 2.

Presenta el hipocono generalmente más largo y ancho que el epicono, el cual tiene una carena puntiaguda y un canal apical lineal corto. El epicono es bilobulado y excavado centralmente en forma de ' $V$ ' invertida. El núcleo está localizado en el hipocono del lado izquierdo, que presenta 2 polos laterales en ambos lados, adyacentes al cíngulo.

Dimensiones: $1 \mathrm{t}=12-18 \mu \mathrm{m}, \mathrm{td}=26-28,6 \mu \mathrm{m}$.

Distribución: se describió por primera vez en Nueva Zelanda, posteriormente reportada para Pacífico Sur y el mar Mediterráneo.

Distribución en México: primer registro para el Pacífico mexicano.

Karenia asterichroma, K. bicuneiformis y K. papilionacea se reportan por primera vez para México. Meave del Castillo \& Hernández-Becerril (1998), mencionaron la presencia de Gymnodinium breve como un registro de literatura para el GT, presumiblemente para referirse a K. asterichroma o K. papilionacea. Los estudios de De Salas et al. (2004) y Haywood et al. (2004) indican que son especies filogenéticamente distintas, aunque sí estrechamente emparentadas. En el presente estudio, la ocurrencia de $K$. papilionacea normalmente está acompañada de $K$. bicuneiformis y a partir de la primera observación en 2009, las 2 especies se han reportado de forma habitual durante la época de secas entre noviembre y mayo, presentándose en densidades bajas de 200 a $400 \mathrm{cel} \mathrm{L}^{-1}$. Karenia bicuneiformis y $K$. papilionacea son especies consideradas como potencialmente tóxicas (Moestrup et al. 2012); Steidinger et al. (2008) indicaron a K. papilionacea como especie productora de brevetoxinas o compuestos similares que fueron determinados por el método ELISA, aunque no ha sido ampliamente documentado, mientras que De Salas et al. (2004), asocia a K. asterichroma con mortalidad de peces en Tasmania.

\section{Familia Polykrikaceae Kofoid et Swezy}

\section{Género Polikrikos Bütschli}

Polykrikos hartmannii Zimmermann (Fig. 3A)

Sinónimos: Phaepolykrikos hartmannii Matsuoka et Fukuyo; Polykrikos barnegatensis Martin
Referencias: Gárate-Lizárraga et al. 2009, p. 21, figs. 30, 31; Hoppenrath et al. 2010, p. 31, fig. 2A-C.

Células coloniales alargadas de forma redondeada en la parte superior y bilobulada en la inferior, presenta 4 zooides, 2 núcleos en la colonia y numerosos cloroplastos.

Dimensiones: $1 \mathrm{t}=77,5 \mu \mathrm{m}, \mathrm{td}=67 \mu \mathrm{m}$.

Distribución: cosmopolita, particularmente en aguas tropicales.

Distribución en México: previamente reportada para el $\mathrm{GC}$, en este estudio se apunta el primer registro para el GT.

En el trabajo de Hoppenrath et al. (2010) hicieron una revisión morfológica y genética de esta especie y propusieron su inclusión dentro del género Polykrikos.

\section{Orden Ptychodiscales Fensome}

Familia Ptychodiscaceae Willey et Hickson

Género BaLechina Loeblich JR. et Loeblich III Balechina coerulea (Dogiel) Taylor (Fig. 3B)

Sinónimo: Gymnodinium coeruleum Dogiel

Referencias: Taylor 1976, p. 113, plate 37, fig. 447, plate 40, fig. 481; Steidinger \& Tangen 1997, p. 461, plate 22.

Célula robusta presenta el epicono cónico redondeado, cingulum excavado descendente, el sulcus se va ensanchando hacia el antapex, núcleo esferoidal situado en el centro, el periplasto que envuelve la célula es fuerte y rígido con estrías longitudinales características del género.

Dimensiones: $1 \mathrm{t}=95 \mu \mathrm{m}, \mathrm{td}=58 \mu \mathrm{m}$.

Distribución: especie costera de aguas cálidas cosmopolita.

Distribución en México: se distribuye a todo lo largo de la costa del Pacífico mexicano.

\section{Orden Noctilucales Haeckel}

Familia Kofoidiniaceae F.J.R Taylor

\section{GÉnero Kofoidinium PaVILLARD}

Kofoidinium pavillardii J. Cachon et M. Cachon (Fig. 3C)

Referencias: Garate-Lizárraga \& Verdugo-Díaz 2007, p. 41, lám. 1, fig. 7; Gómez \& Furuya 2007, p. 118, figs. 8-9 y 1215. 


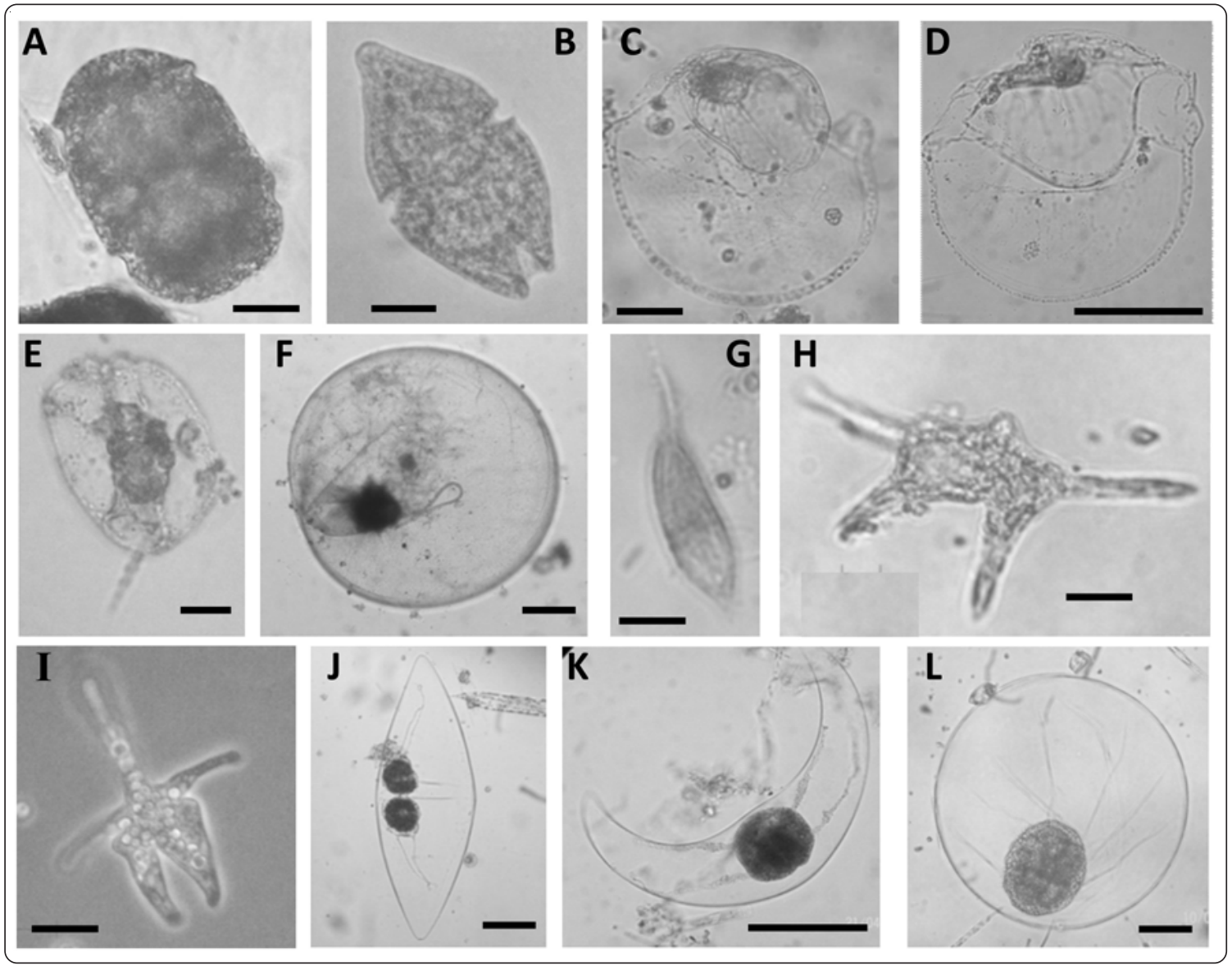

Figura 3. Especies de dinoflagelados atecados de costas de Chiapas, México, microscopía de luz. A) Polykrikos hartmannii, B) Balechina coerulea, C) Kofoidinium pavillardii, D) Kofoidinium splendens, E) Spatulodinium pseudonoctiluca, F) Noctiluca scintillans, G) Pronoctiluca spinifera, H) Brachidinium capitatum, I) Asterodinium gracile, J) Pyrocystis fusiformis, K) Pyrocystis lunula, L) Pyrocystis noctiluca. Escalas en Fig. G representa $10 \mu \mathrm{m}$, Figs. A-B, E, H, I representan $20 \mu \mathrm{m}$, y Figs. C, D, F, J-L representan $100 \mu \mathrm{m} /$ Species of athecate dinoflagellates from the coasts of Chiapas, Mexico, light microscopy. A) Polykrikos hartmannii, B) Balechina coerulea, C) Kofoidinium pavillardii, D) Kofoidinium splendens, E) Spatulodinium pseudonoctiluca, F) Noctiluca scintillans, G) Pronoctiluca spinifera, H) Brachidinium capitatum, I) Asterodinium gracile, J) Pyrocystis fusiformis, K) Pyrocystis lunula, L) Pyrocystis noctiluca. Scales in Fig. G represents $10 \mu \mathrm{m}$, Figs. A-B, E, H, I represent $20 \mu \mathrm{m}$, and Figs. C-D, F, J-L represent $100 \mu \mathrm{m}$

De gran tamaño, la mayor parte del cuerpo es de forma aplanada semejando un plato, es la especie más grande del género con diámetros mayores a $300 \mu \mathrm{m}$, la característica predominante es la saliente cuadrada como se aprecia claramente en la figura de este estudio.

Dimensiones: $\mathrm{d}=312 \mu \mathrm{m}$.

Distribución: registrada para el mar Mediterráneo y el Pacífico oriental y occidental.

Distribución en México: ha sido reportada para el GC y las costas de Guerrero, en este estudio se menciona la ampliación de ámbito al GT.
Kofoidinium splendens J. Cachon et M. Cachon (Fig. 3D)

Referencias: Taylor 1976, p. 185, plate 39, figs. 475, 476; Gómez \& Furuya 2007, p. 118, fig. 16.

Cuerpo aplanado, reforzado por formaciones fibrilares, episoma de forma redondeada, presenta cuerpos de inclusión de coloración rojiza característica de la especie, es la especie con dimensiones más pequeñas.

Dimensiones: 2 ejemplares, $\mathrm{d}=169-180 \mu \mathrm{m}$.

Distribución general: nerítica de aguas templadas y tropicales. 
Distribución en México: en este estudio se da la primera mención de esta especie para el GT.

Las especies del género Kofoidinium presentan características muy semejantes, así que Gómez \& Furuya (2007) han propuesto diferenciarlas de acuerdo al tamaño y la presencia de proyecciones en el episoma y el hiposoma. K. pavillardi es la especie de mayor tamaño y presenta una proyección conocida como saliente cuadrada (Fig. 3C), estructura de la cual carece $K$. splendens. En la costa de Chiapas estas 2 especies se presentan en la temporada de vientos 'tehuanos', respondiendo a la hipótesis que las especies del cnefoplancton (plancton de penumbra) son arrastradas hacia la costa por eventos de surgencia.

\section{Género Spatulodinium Cachon et Cachon}

Spatulodinium pseudonoctiluca (Pouchet) Cachon et Cachon ex Loeblich Jr. et Loeblich III (Fig. 3E)

Sinónimo: Gymnodinium pseudonoctiluca Pouchet; Gymnodinium fulgens Kofoid et Swezy

Referencias: Dodge 1982, p. 136, fig. 16D; Avancini et al. 2006, p. 413, figs. A-C; Gómez et al. 2010, p. 468, figs. MQ; Gárate-Lizárraga 2011, p. 35, figs. 11-14.

Considerada actualmente como la única especie del género, presenta un complejo ciclo de vida con varios estadios inmaduros tipo gymnodinoides (que en su momento fueron identificados como especies diferentes, como Gymnodinium lebouriae); las células maduras presentan forma ovalada con un tentáculo rígido.

Dimensiones: $1 \mathrm{t}=120 \mu \mathrm{m}, \mathrm{td}=95 \mu \mathrm{m}$.

Distribución: inicialmente reportada en el Mediterráneo y mar de Japón, posteriormente en el Pacífico sudamericano.

Distribución en México: reportada en el GC hasta Salina Cruz, Oaxaca.

Gárate-Lizárraga (2011) estableció la distribución de Spatulodinum pseudonoctiluca, en el litoral del Pacífico mexicano, desde el GC hasta Salina Cruz Oaxaca, indicando los estadios de vida, en este estudio se reporta la ampliación de ámbito de la especie documentando el estadio adulto. Los estadios juveniles no se observaron en las muestras estudiadas.
Clase Noctiluciphyceae Fensome et al.

Orden Noctilucales Haeckel

Familia Noctilucaceae SaVille-Kent

\section{GÉNERo NoctILUCA SURIRAY}

Noctiluca scintillans (Macartney) Kofoid et Swezy (Fig. 3F)

Sinónimos: Medusa scintillans Macartney; Noctiluca miliaris Suriray ex Lamarck

Referencias: Steidinger \& Tangen 1997, p. 466, plate 23; Avancini et al. 2006, p. 361, fig. A-C; Esqueda-Lara \& Hernández-Becerril 2010, p. 179, figs. 172a-b.

El cuerpo globular y grande, no se distingue el epicono del hipocono, el sulcus termina atrás del tentáculo el cual es muy desarrollado y con estrías transversales muy visibles, cloroplastos ausentes, el núcleo se encuentra en el extremo próximo al tentáculo

Dimensiones: $\mathrm{d}=480 \mu \mathrm{m}$.

Distribución: cosmopolita de aguas cálidas

Distribución en México: se ha reportado en todo el Pacífico mexicano.

Noctiluca scintillans es una especie común en todos los mares del mundo como formadora de mareas rojas, en este estudio fue frecuente, aunque en densidades bajas.

\section{Género Pronoctiluca Fabre-Domergue}

Pronoctiluca spinifera (Lohmann) Schiller (Fig. 3G)

Referencias: Taylor 1976, p. 188, plate 37, fig. 429; Dodge, 1982 p. 112, fig. 13G.

Célula de forma fusiforme, como característica distintiva presenta 2 extensiones, una proyección apical en forma de tentáculo, y otra en el extremo opuesto, núcleo relativamente grande que abarca el hiposoma, no presenta cloroplastos.

Dimensiones: $1 \mathrm{t}=25 \mu \mathrm{m}, \mathrm{td}=12 \mu \mathrm{m}$.

Distribución: de amplia distribución, sin embargo no se observa frecuentemente.

Distribución en México: reportada previamente para el GC. 


\section{Orden Brachidiniales Loeblich III ex Sournia}

Familia Brachidiniaceae Sournia

\section{GÉNERo BRACHIDINIUM F.J.R. TAYLOR}

Brachidinium capitatum F.J.R. Taylor (Fig. 3H)

Referencias: Hernández-Becerril \& Bravo-Sierra 2004, p. 419, figs. 10, 11; Gómez et al. 2005b, p. 266, figs. 16, 18 ; Gárate-Lizárraga \& Verdugo-Díaz 2007, p. 40. lám. 1, fig. 3.

Célula aplanada con 4 extensiones partiendo del hipocono y un proceso apical en el epicono, el núcleo es grande y ocupa la mayor parte del cuerpo hacia el lado izquierdo. Los brazos superiores son más largos que los posteriores.

Dimensiones: $1 \mathrm{t}=51,9 \mu \mathrm{m}, \mathrm{td}=42,6 \mu \mathrm{m}$, largo total de los brazos extendidos $=107,5 \mu \mathrm{m}$.

Distribución general: inicialmente descrita para el océano Indico, reportada para Japón y el Pacífico oriental en las costas de Perú.

Distribución en México: registrada por primera vez para el Pacífico mexicano en el área de Bahía Magdalena, GC. Primer registro para el GT.

Las especies del género Brachidinium han sido cuestionadas por Gómez et al. (2005b), ya que B. catenatum y $B$. brevipes no han sido reportadas desde su descripción original, y sugirieron que son variantes de la especie tipo y de la incorporación de este género a Karenia, debido principalmente a las semejanzas morfológicas con Karenia papilionacea, además los autores han llegado a sugerir que Brachidinium es una fase del ciclo de vida de ésta. Henrichs et al. (2011) encontraron que B. capitatum se encuentra más relacionada filogenéticamente con $K$. bicuneiformis que con $K$. papilionacea, sin embargo se requieren otros estudios para poder sugerir su inclusión en Kareniaceae. A pesar de observarse en cantidades pequeñas, B. capitatum es capaz de producir proliferaciones y las células en crecimiento pueden ser transportadas incluso a los estuarios (Henrichs et al. 2011).

\section{GÉNERo Asterodinium SOURNIA}

Asterodinium gracile Sournia (Fig. 3I)

Referencias: Gómez 2003, p. 334, figs. 3-12; Gómez et al. 2005b, p. 265, fig. 23; Gárate-Lizárraga \& Verdugo-Díaz 2007, p. 39, lám. 1, figs. 1, 2.

Presenta el cuerpo dorsoventralmente aplanado, con 2 extensiones en el hiposoma y 3 en el episoma, núcleo bien desarrollado del lado izquierdo del hipocono con la presencia de numerosos cloroplastos.

Dimensiones: $1 \mathrm{t}=98,3 \mu \mathrm{m}, \mathrm{td}=33,2$, separación máxima de brazos laterales $=79,5 \mu \mathrm{m}$, longitud del proceso apical $=47,4 \mu \mathrm{m}$.

Distribución general: amplia distribución, sin embargo existen pocos reportes.

Distribución en México: reportada para el GC, el ejemplar en este estudio, corresponde al registro más austral de esta especie, ampliando así su rango de distribución en el Pacífico mexicano.

Asterodinium gracile pertenece a un grupo de especies oceánicas de aguas profundas que forman parte del cnefoplancton (plancton de penumbra), su presencia no ha sido frecuente en todas las muestras, lo que sugiere que su presencia en las muestras costeras puede ser debida a surgencias provocadas por el efecto de los vientos 'tehuanos' Gómez (2006) expresó que los registros menos profundos coinciden con la presencia de Brachidinium capitatum, como ocurre en este estudio.

\section{Orden Pyrocystales}

\section{Familia Pyrocystaceae Apstein}

\section{GÉnero Pyrocystis Murray}

Pyrocystis fusiformis (Wyville-Thomson ex Haeckel) Blackman (Fig. 3J)

Referencias: Balech 1988, p. 25, lám. 2, figs. 9, 10; GárateLizárraga \& Verdugo-Díaz 2007, p. 40, lám. 1, fig. 6; Esqueda-Lara \& Hernández-Becerril 2010, p. 100, fig. 93.

Muy grande, con los extremos agudos a ligeramente redondeados, presenta variaciones bastante amplias en cuanto a dimensiones, algunos ejemplares parecen más alargados y otros tienden a ser más redondos, según Balech (1988) se trata de la misma especie y las diferencias individuales representan distintos estadios de desarrollo, puede presentar hasta 2 estadios gymnodinoides concentrados en el centro de la estructura desde donde parten unas proyecciones.

Dimensiones: $1 \mathrm{t}=450 \mu \mathrm{m}, \mathrm{td}=170 \mu \mathrm{m}$.

Distribución: cosmopolita de aguas tropicales y subtropicales.

Distribución en México: ampliamente distribuida en el Pacífico mexicano. 
Pyrocystis lunula (Schütt) Schütt (Fig. 3K)

Sinónimo: Gymnodinium lunula Schütt; Dissodinium lunula (Schütt) Klebs in Pascher

Referencias: Balech 1988, p. 24, lám. 2, figs. 3, 4; EsquedaLara \& Hernández-Becerril 2010, p. 101, fig. 94.

Quistes semilunares de curvatura moderada y extremos bien separados, protoplasma acumulado en la parte central de donde parten las proyecciones hacia el extremo de la estructura.

Dimensiones: $1 \mathrm{t}=170 \mu \mathrm{m}$.

Distribución: presente en aguas costeras templadas y tropicales.

Distribución en México: reportada previamente del GC hasta las costas de Jalisco y Colima.

Pyrocystis noctiluca Murray ex Haeckel (Fig. 3L)

Referencias: Balech 1988, p. 24, lám. 2, fig. 2 GárateLizárraga 2008, p. 62, fig. 24, Esqueda-Lara \& HernándezBecerril 2010 p. 102, fig. 95.

De forma esférica y grande, protoplasma concentrado en una masa pequeña que incluye el núcleo y de la que parten cordones irregulares periféricos.

Dimensiones: $\mathrm{d}=625 \mu \mathrm{m}$.

Distribución: especie de amplia distribución se presenta tanto en aguas templadas como tropicales.

Distribución en México: se ha registrado lo largo de la costa del Pacífico.

\section{Discusión}

La taxonomía de los dinoflagelados desnudos es una tarea complicada, debido principalmente a la dificultad que supone el estudio taxonómico de un grupo tan polimórfico, con relativamente pocos caracteres morfológicos y con una variabilidad intraespecífica difícil de apreciar en la mayoría de los casos (e.g., Larsen 2002). Se conocen algunas especies que muestran un cierto grado de variabilidad morfológica posiblemente relacionado con el ambiente, mientras que en otros casos las especies se mantienen en un rango de variabilidad estrecho, sin mostrar aparentemente ninguna relación con el ambiente. Adicionalmente, los agentes conservantes empleados rutinariamente modifican los caracteres morfológicos.

En este estudio se describen e ilustran 27 especies de dinoflagelados atecados para las costas de Chiapas, de las cuales 3 especies constituyen nuevos registros para el Pacífico mexicano: Cochlodinium pulchellum, Karenia bicuneiformis $(=K$. bidigitata) y K. papilionacea. Este número de especies encontradas puede ser considerado alto, sobre todo debido a que el método de recolección por red puede causar sesgo en las especies frágiles o pequeñas. Okolodkov \& Gárate-Lizárraga (2006) listaron 64 especies de dinoflagelados atecados hasta el 2005 para el Pacífico mexicano, de las cuales únicamente 15 especies se citan para el golfo de Tehuantepec, la gran mayoría (12) son listadas en el trabajo de Meave del Castillo \& Hernández-Becerril (1998). En un trabajo reciente, Meave del Castillo et al. (2012) detectaron 32 nuevos registros de dinoflagelados desnudos en la zona de Acapulco, Pacífico central mexicano.

Los resultados de este trabajo concuerdan con los autores anteriores únicamente con 4 taxa: Balechina coerulea, Gyrodinium falcatum, Noctiluca scintillans y Pyrocystis fusiformis, quizás debido a los diferentes métodos de estudio, principalmente de recolección y preservación (utilización de red, uso de formaldehído, etc.). Pyrocystis noctiluca ha sido previamente citado por Gárate-Lizárraga (2008) para el golfo de Tehuantepec.

En relación con estudios de otras áreas del Pacífico mexicano, destaca la similitud en número de especies estudiadas por Gárate-Lizárraga et al. (2007) en el sistema lagunar Magdalena-Almejas, Baja California Sur, que coincide en 14 taxa; en el golfo de California, Licea et al. (1995) incluyeron 25 especies de dinoflagelados atecados, que coincide con 7 especies con el presente estudio, principalmente con especies del género Pyrocystis y formadoras de mareas rojas y casi todas los registros hechos por Gárate-Lizárraga \& Verdugo-Díaz (2007) en esa región, también son consignados aquí, mientras que Gárate-Lizárraga et al. (2009) y Meave del Castillo et al. (2012) listaron e ilustraron varias especies que no se han detectado en este estudio, entre ellas especies de los géneros Amphidinium, Cochlodinium, Gymnodinium, Gyrodinium, Katodinium, Polykrikos, Takayama y Warnowia.

El número de especies reportados parece ser pequeño en relación a las 470 especies de dinoflagelados atecados descritos a nivel mundial listadas por Gómez (2005), sin embargo los resultados son ampliamente significativos ya que representan en número el $42 \%$ de especies reportadas por Okolodkov \& Gárate-Lizárraga (2006) y se suman 23 especies a las reportadas para el golfo de Tehuantepec por Meave \& Hernández-Becerril (1998), 
además que Cochlodinium helicoides y Amphidinium cf. operculatum son citados por segunda vez. Asímismo, es importante mencionar que este es el primer trabajo que describe e ilustra especies de dinoflagelados atecados exclusivamente para el estado de Chiapas, de tal modo que se amplía el rango de distribución de los taxa encontrados en este estudio.

La presencia de nuevos registros puede estar realcionada con la falta de investigación en ciertas áreas, principalmente el Pacífico tropical mexicano, quizás se ha subestimado la presencia de ciertas formas de dinoflagelados desnudos ya sea ignorándolos o con identificación errónea. Aunque la introducción de especies exóticas es un tema relacionado a los nuevos registros de especies y puede originar especulaciones, se necesita contar con datos históricos, que no existen para la mayoría de las regiones del país, o muestras especializadas (e.g., sedimentos geológicos) que puedan ser estudiadas de manera adecuada.

El inventario de dinoflagelados desnudos del estado de Chiapas y en el país, es todavía incompleto y se requieren de estudios más detallados y específicos para este grupo utilizando métodos de recolección y preservación distintos a los tradicionales, que permitan que las especies de este grupo estén bien representadas y no se deformen, al mismo tiempo se necesita mayor continuidad. La aplicación de nuevas técnicas que complementen el análisis morfológico (e.g., estudios de biología molecular), a la par con descripciones de aspectos ecológicos y desarrollo de cultivos, podrán incrementar el número de especies conocidas en esta zona del Pacífico mexicano.

\section{Agradecimientos}

A los compañeros de las Jurisdicciones Sanitarias VII y VIII por su ayuda en la toma de muestras y a la QFB. Adriana Gómez Bustamante, directora del 'Laboratorio Estatal de Salud Pública' del estado de Chiapas, por las facilidades otorgadas en la realización de este trabajo.

\section{LITERATURA CITADA}

Alonso-Rodríguez R, DU Hernández-Becerril \& I GárateLizárraga. 2008. Catálogo de microalgas de las lagunas costeras de Sinaloa. En: Páez-Osuna F (ed). Serie lagunas costeras de Sinaloa 4: 1-198. Instituto de Ciencias del Mar y Limnología, Universidad Nacional Autónoma de México, México.
Avancini M, AM Cicero, I Di Girolamo, M Innamorati, E Magaletti, T Sertorio \& T Zunini. 2006. Guida al riconoscimento del plancton dei mari italiani, Vol. I. Fitoplancton, 503 pp. Ministero dell'Ambiente e della Tutela del Territorio e del Mare. ICRAM, Roma.

Balech E. 1988. Los dinoflagelados del Atlántico Sudoccidental, 219 pp. Publicación Especial, Instituto Español de Oceanografía, Madrid.

Botes L, SD Sym \& GC Pitcher. 2003. Karenia cristata sp. nov. and Karenia bicuneiformis sp. nov. (Gymnodiniales Dinophyceae): two new Karenia species from the South Africa coast. Phycologia 42: 563-571.

De Salas MF, CJS Bolch \& GM Hallegraeff. 2004. Karenia asterichroma sp. nov. (Gymnodiniales, Dinophyceae) a new dinoflagellate species associated with finfish aquaculture mortalities en Tasmania Australia. Phycologia 43: 624-631.

Dodge JD. 1982. Marine dinoflagellates of the British Isles, 303 pp. Govt. Bookshops, Londres.

Esqueda-Lara K \& DU Hernández-Becerril. 2010. Dinoflagelados microplanctónicos marinos del Pacífico central de México (Isla Isabel, Nayarit y costas de Jalisco y Colima, 206 pp. Instituto de Ciencias del Mar y Limnología, Universidad Nacional Autónoma de México, México.

Fensome RA, FJR Taylor, G Norris, WAS Sarjeant, DI Wharton \& GL Williams. 1993. A classification of fossil and living dinoflagellates. Micropaleontology Press Special Paper 7: 1-351.

Gárate-Lizárraga I. 2008. Nuevos registros de dinoflagelados planctónicos para el Golfo de Tehuantepec, México. CICIMAR Oceánides 23: 55-65.

Gárate-Lizárraga I. 2011. New data on the distribution of Spatulodinium pseudonoctiluca (Noctilucales: Kofoidiniaceae) in the Mexican Pacific. CICIMAR Oceánides 26: 33-41.

Gárate-Lizárraga I \& G Verdugo-Díaz. 2007. Nuevos registros de dinoflagelados desnudos para el Golfo de California, México. CICIMAR Oceánides 22: 37-43.

Gárate-Lizárraga I, DJ López-Cortes, JJ BustillosGúzman \& F Hernández-Sandoval. 2004. Blooms of Cochlodinium polikrikoides (Gymnodiniaceae) in the Gulf of California, México. Revista de Biología Tropical 52 (Suppl. 1): 51-58.

Gárate-Lizárraga I, CJ Band-Schmidt, G Verdugo-Díaz, MS Muletón-Gómez \& EF Félix-Pico. 2007. Dinoflagelados (Dinophyceae) del sistema lagunar Magdalena-Almejas. En: Funes-Rodríguez R, J Gómez-Gutiérrez \& R PalomaresGarcía (eds). Estudios ecológicos en Bahía Magdalena, pp. 145-174. Centro Interdisciplinario de Ciencias MarinasInstituto Politécnico Nacional, La Paz.

Gárate-Lizárraga I, CJ Band-Schmidt, F Aguirre Bahena \& T Grayeb-del Alamo. 2009. A multi-species microalgae bloom in Bahia de La Paz, Gulf of California, Mexico (June 2008). CICIMAR Oceánides 24:15-29. 
Gárate-Lizárraga I, E Muciño-Márquez \& DJ LópezCortés. 2010. Estadios de vida de Gyrodinium falcatum (Dinophyceae) en la Bahía de La Paz, Golfo de California. CICIMAR Oceánides 25: 53-58.

Gárate-Lizárraga I, F García-Domínguez, B Pérez-Cruz \& JA Díaz-Ortiz. 2011. First record of Cochlodinium convolutum and C. helicoides (Gymnodiniales: Dinophyceae) in the Gulf of California. Revista de Biología Marina y Oceanografía 46: 495-498.

Gómez F. 2003. New records of Asterodinium Sournia (Brachidiniales, Dinophyceae). Nova Hedwigia 77: 331340 .

Gómez F. 2005. A list of free-living dinoflagellate species in the world's oceans. Acta Botanica Croatica 64: 129-212.

Gómez F. 2006. The dinoflagellate genera Brachidinium, Asterodinium, Microceratium and Karenia in the open SE Pacific Ocean. Algae 21: 445-452.

Gómez F. 2007a. Gynogonadinium aequatoriale gen. et sp. nov., a new dinoflagellate from the open western equatorial pacific. Algae 22: 11-15.

Gómez F. 2007b. Gymnodinioid Dinoflagellates (Gymnodiniales, Dinophyceae) in the Open Pacific Ocean. Algae 22: 273-286.

Gómez F. 2009. Torodinium and Pavillardia (Gymnodiniales, Dinophyceae): two unarmoured dinoflagellates with a body extension, collected from the open Pacific Ocean. Protistology 6: 131-135.

Gómez F \& K Furuya. 2007. Kofoidinium, Spatulodinium and other kofoidiniaceans (Noctilucales, Dinophyceae) in the Pacific Ocean. European Journal of Protistology 43: 115124.

Gómez F, S Yoshimatsu \& K Furuya. 2005a. Morphology of Brachidinium capitatum FJR Taylor (Brachidiniales, Dinophyceae) collected from the western Pacific Ocean. Cryptogamie Algologie 26: 165-175.

Gómez F, Y Nagahama, H Takayama \& K Furuya. 2005b. Is Karenia a synonym of Asterodinium-Brachidinium (Gymnodiniales, Dinophyceae)? Acta Botanica Croatica 64: 263-274.

Gómez F, D Moreira \& P López-García. 2010. Molecular phylogeny of Noctilucoid dinoflagellates (Noctilucales, Dinophyceae). Protist 161: 466-478.

Hallegraeff GM. 2002. Aquaculturists' guide to harmful Australian microalgae, $136 \mathrm{pp}$. University of Tasmania, Hobart.

Haywood AJ, KA Steidinger, EW Truby, PR Bergquist, PL Bergquist, J Adamson \& L MacKenzie. 2004. Comparative morphology and molecular phylogenetic analysis of three new species of the genus Karenia (Dinophyceae) from New Zealand. Journal of Phycology 40: 165-179.
Henrichs DW, HM Sosik, RJ Olson \& L Campbell. 2011. Phylogenetic analysis of Brachidinium capitatum (Dinophyceae) from the Gulf of Mexico indicates membership in the kareniaceae. Journal of Phycology 47: 366-374.

Hernández-Becerril DU \& E Bravo-Sierra. 2004. New records of planktonic dinoflagellates (Dinophyceae) from the Mexican Pacific Ocean. Botanica Marina 47: 417-423.

Hoppenrath M, N Yubuki, TN Bachvaroff \& BS Leander. 2010. Re-classification of Pheopolykrikos hartmannii as Polykrikos (Dinophyceae) based partly on the ultrastructure of complex extrusomes. European Journal of Protistology 46: $29-37$

Iwataki M, H Kawami \& K Matzuoka. 2007. Cochlodinium fulvescens sp. nov. (Gymnodiniales, Dinophyceae), a new chain-forming unarmored dinoflagellate from Asian coasts. Phycological Research 55:231-239.

Jørgensen MF, S Murray \& N Daugbjerg. 2004. Amphidinium revisited. I. Redefinition of Amphidinium (Dinophyceae) based on cladistic and molecular phylogenetic analyses. Journal of Phycology 40: 351-365.

Konovalova GV. 2003. The life history of Gyrodinium falcatum and validity of Pseliodinium vaubanii (Dinophyceae). Russian Journal of Marine Biology 29: 167-170.

Larsen J. 2002. Dinoflagelados atecados pot.encialmente toxígenos en el cono sur americano. En: Sar EA, ME Ferrario \& B Reguera (eds). Floraciones algales nocivas en el cono sur americano, pp. 147-154. Instituto Español de Oceanografía, Madrid.

Licea S, JL Moreno, H Santoyo \& G Figueroa. 1995. Dinoflageladas del Golfo de California, 165 pp. Universidad Autónoma de California Sur, La Paz.

Matsuoka K, I Mitsunori \& H Kawami. 2008. Morphology and taxonomy of chain-forming species of the genus Cochlodinium (Dinophyceae). Harmful Algae 7: 261-270.

Meave del Castillo ME \& DU Hernández-Becerril. 1998. Fitoplancton. En: Tapia-García M (ed). El Golfo de Tehuantepec: el ecosistema y sus recursos, pp. 59-74. Universidad Autónoma Metropolitana Iztapalapa, México.

Meave del Castillo ME, ME Zamudio-Resendiz \& M Castillo-Rivera. 2012. Riqueza fitoplanctónica de la Bahía de Acapulco y zona costera aledaña, Guerrero, México. Acta Botánica Mexicana 100: 405-487.

Moestrup Ø, R Akselman, G Cronberg, M Elbraechter, S Fraga, Y Halim, G Hansen, M Hoppenrath, J Larsen, N Lundholm, LN Nguyen \& A Zingone. 2012. Taxonomic reference list of harmful microalgae, IOC-UNESCO. $<$ http:/ /www.marinespecies.org/HAB>

Monreal MA \& DA Salas de León. 1998. Dinámica y estructura termohalina. En: Tapia-García M (ed). El Golfo de Tehuantepec: el ecosistema y sus recursos, pp. 13-26. Universidad Autónoma Metropolitana Iztapalapa, México, D.F. 
Murray S, MF Jørgensen, N Daugbjerg \& L Rhodes. 2004. Amphidinium revisited. II. Resolving species boundaries in the Amphidinium operculatum species complex (Dinophyceae), including the descriptions of Amphidinium trulla sp. nov. and Amphidinium gibbosum. comb. nov. Journal of Phycology 40: 366-382.

Núñez-Vázquez EJ. 2005. Biotoxinas marinas en peces comestibles de Baja California Sur, México: origen, identificación y cuantificación. Tesis de Biología Marina, Área Interdisciplinaria de Ciencias del Mar, Departamento de Biología Marina, Universidad Autónoma de Baja California Sur, La Paz, 87 pp.

Okolodkov YB \& I Gárate-Lizárraga. 2006. An annotated checklist of dinoflagellates (Dinophyceae) from the Mexican Pacific. Acta Botánica Mexicana 74: 1-154.

Sournia A. 1972. Une période de poussées phytoplanctoniques prés de Nosy-Bé (Madagascar) en 1971. I. Espèces rares ou nouvelle du phythoplancton. Cahiers ORSTOM. Série Océanographie 10(2): 151-159.

Steidinger KA \& K Tangen. 1997. Dinoflagellates. In: Tomas CR (ed). Identifying marine phytoplankton, pp. 387-584. Academic Press, San Diego.
Steidinger KA, JL Wolny \& AJ Haywood. 2008. Identification of Kareniaceae (Dinophyceae) in the Gulf of Mexico. Nova Hedwigia 133: 269-284.

Taylor FJR. 1976. Dinoflagellates from the International Indian Ocean Expedition. A report on material collected by the R. V. “Anton Bruun" 1963-1964. Bibliotheca Botanica 132: 1-234, pl. 1-46.

Taylor FJR, M Hoppenrath \& JF Saldarriaga. 2008. Dinoflagellate diversity and distribution. Biodiversity Conservation 17: 407-418.

Trasviña A \& ED Burton. 1997. Los 'Nortes' del golfo de Tehuantepec la circulación costera inducida por el viento. En: Lavin MF (ed). Contribuciones a la oceanografía física en México. Monografia 3: 25-46. Unión Geofísica Mexicana, México, D.F.

Uchida T, Y Matsuyama, M Yamaguchi \& T Honjo. 1996. The life cycle of Gyrodinium instriatum (Dinophyceae) in culture. Phycological Research 44: 119-123.

Vargas-Montero M, F Bustamante, JC Gúzman \& JC Vargas. 2008. Florecimientos de dinoflagelados nocivos en la costa Pacífica de Costa Rica. Hidrobiológica 18 (Supl. 1): $15-23$.

Recibido el 11 de junio de 2012 y aceptado el 19 de abril de 2013

Editor Asociado: Pilar Muñoz M. 